\title{
Altered Brain Activation During Memory Retrieval Precedes and Predicts Conversion to Psychosis in Individuals at Clinical High Risk
}

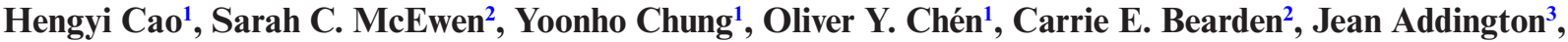 \\ Bradley Goodyear ${ }^{3-5}$, Kristin S. Cadenhead ${ }^{6}$, Heline Mirzakhanian ${ }^{6}$, Barbara A. Cornblatt ${ }^{7}$, Ricardo E. Carrión ${ }^{7}$, \\ Daniel H. Mathalon ${ }^{8}$, Thomas H. McGlashan', Diana O. Perkins ${ }^{10}$, Aysenil Belger ${ }^{10}$, Larry J. Seidman ${ }^{11}$, \\ Heidi Thermenos ${ }^{11}$, Ming T. Tsuang ${ }^{6}$, Theo G. M. van Erp ${ }^{12}$, Elaine F. Walker ${ }^{13}$, Stephan Hamann ${ }^{13}$, Alan Anticevic ${ }^{9}$, \\ Scott W. Woods ${ }^{9}$, and Tyrone D. Cannon*,1,9

\begin{abstract}
${ }^{1}$ Department of Psychology, Yale University, New Haven, CT; ${ }^{2}$ Department of Psychiatry and Biobehavioral Sciences, University of California Los Angeles, Los Angeles, CA; ${ }^{3}$ Department of Psychiatry, University of Calgary, Calgary, Canada; ${ }^{4}$ Department of Radiology, University of Calgary, Calgary, Canada; ${ }^{5}$ Department of Clinical Neuroscience, University of Calgary, Calgary, Canada; ${ }^{6}$ Department of Psychiatry, University of California San Diego, San Diego, CA; ${ }^{7}$ Department of Psychiatry Research, Zucker Hillside Hospital, Glen Oaks, NY; ${ }^{8}$ Department of Psychiatry, University of California San Francisco, San Francisco, CA; ${ }^{9}$ Department of Psychiatry, Yale University, New Haven, CT; ${ }^{10}$ Department of Psychiatry, University of North Carolina, Chapel Hill, Chapel Hill, NC; ${ }^{11}$ Department of Psychiatry, Beth Israel Deaconess Medical Center, Harvard Medical School, Boston, MA; ${ }^{12}$ Department of Psychiatry
\end{abstract} \\ and Human Behavior, University of California Irvine, Irvine, CA; ${ }^{13}$ Department of Psychology, Emory University, Atlanta, GA
}

*To whom correspondence should be addressed; Department of Psychology, Yale University, 2 Hillhouse Avenue, New Haven, CT 06511, US; tel: +1-2034361545, e-mail: tyrone.cannon@yale.edu

Memory deficits are a hallmark of psychotic disorders such as schizophrenia. However, whether the neural dysfunction underlying these deficits is present before the onset of illness and potentially predicts conversion to psychosis is unclear. In this study, we investigated brain functional alterations during memory processing in a sample of $\mathbf{1 5 5}$ individuals at clinical high risk (including 18 subjects who later converted to full psychosis) and 108 healthy controls drawn from the second phase of the North American Prodrome Longitudinal Study (NAPLS-2). All participants underwent functional magnetic resonance imaging with a paired-associate memory paradigm at the point of recruitment and were clinically followed up for approximately 2 years. We found that at baseline, subjects at high risk showed significantly higher activation during memory retrieval in the prefrontal, parietal, and bilateral temporal cortices $\left(P_{\mathrm{FWE}}<.035\right)$. This effect was more pronounced in converters than nonconverters and was particularly manifested in unmedicated subjects $(P<.001)$. The hyperactivation was significantly correlated with retrieval reaction time during scan in converters $(P=.009)$ but not in nonconverters and controls, suggesting an exaggerated retrieval effort. These findings suggest that hyperactivation during memory retrieval may mark processes associated with conversion to psychosis, and such measures have potential as biomarkers for psychosis prediction.

Key words: fMRI/associative memory/memory retrieval/psychosis/clinical high risk

\section{Introduction}

Memory impairments are among the most robust correlates of schizophrenia and related psychotic disorders. ${ }^{1-3}$ Prior work has shown that such deficits are present before the occurrence of clinical symptoms ${ }^{46}$ and are significantly more severe in prodromal subjects who later convert to full psychosis than those who do not. ${ }^{7,8}$ Nevertheless, whether brain physiological measures during memory processing are predictive of future psychotic disorders in individuals at risk remains to be determined.

Compared to memory for single items, episodic memory processing for associated items seems to be particularly impaired in patients with schizophrenia. ${ }^{2}$ Neuroimaging studies have demonstrated that the neural substrates of associative memory involve multiple regions including the dorsolateral prefrontal cortex (DLPFC), ventrolateral prefrontal cortex (VLPFC), posterior parietal cortex, and medial temporal cortex. ${ }^{9-12}$ Dysfunction in these regions has been widely reported in patients with psychotic disorders, in particular schizophrenia., ${ }^{3,6,13-20}$ Such dysfunction involves altered prefrontal, parietal, and temporal cortical reactivity ${ }^{3,14-20}$ and aberrant functional connectivity in the prefrontal and temporal cortex, ${ }^{6,13}$ during both memory encoding and memory retrieval. In addition, evidence has shown that these functional alterations may further lead to the reorganization of brain network architecture in patients during memory 
and cognition, in particular deficits in measures of network efficiency and network segregation. . $^{21,22}$ Notably, it has been demonstrated that neural dysfunction during memory processing cannot be fully explained by the severity of clinical symptoms ${ }^{17}$ nor medication status, ${ }^{23}$ and can also be detected in unaffected first-degree relatives of patients with schizophrenia, ${ }^{18,24-27}$ suggesting that it occurs before the onset of illness. Nevertheless, it remains unclear whether physiological alterations during memory encoding or retrieval are present in individuals at clinical high risk (CHR) and whether such alternations predict conversion to psychosis.

Here, we used functional magnetic resonance imaging (fMRI) during a paired-associate learning and memory task to investigate potential brain activation and network alterations predictive of psychosis in a sample of 155 CHR subjects (including 18 cases who converted to psychosis) and 108 healthy controls (HCs) drawn from the second phase of the North American Prodrome Longitudinal Study (NAPLS-2). ${ }^{28}$ Specifically, we tested whether subjects at CHR show abnormal neural response during the encoding and/or retrieval phases of the memory task, and whether such baseline abnormalities can potentially predict future psychosis. Moreover, because we have previously reported resting-state network efficiency and network diversity as potential biomarkers for psychosis development, ${ }^{29}$ we further used a graphbased analysis to examine whether subjects at CHR deviate from HCs on these metrics during the memory task. We hypothesized that similar to patients with psychosis, subjects at CHR may show dysfunction in regions that are pertinent to memory processing during the associative memory task, in particular prefrontal, parietal, and temporal regions, and such changes may particularly be present in those who eventually convert to full psychosis.

\section{Methods and Materials}

\section{Subjects}

Subjects in this study were drawn from the NAPLS-2 project, ${ }^{28}$ which included 8 study sites across the United States and Canada. Because fMRI paradigms were introduced half way through the NAPLS-2 project, the present report consisted of 263 subjects with fMRI data available at the initial recruitment point after quality control (155 subjects at CHR, including 18 subjects who converted into psychosis during follow-up, and $108 \mathrm{HCs}$, see supplementary material for details). The general exclusion criteria included a prior history of neurological disorders, substance dependence in the last 6 months, and intelligence quotient $<70$ (assessed by the Wechsler Abbreviated Scale of Intelligence ${ }^{30}$ ). The CHR subjects met the Structured Interview for Prodromal Syndromes (SIPS) criteria of prodromal syndromes ${ }^{31}$ at baseline after excluding individuals who had ever met DSM-IV (Diagnostic and Statistical Manual of Mental Disorders,
Fourth Edition) criteria for a psychotic disorder. ${ }^{32}$ The converters met the criteria for an Axis-I psychotic disorder or with the presence of at least one fully psychotic symptom at follow-up. HCs were excluded if they met the criteria for a psychotic disorder or prodromal syndrome, or had a first-degree relative with mental illness. All participants provided written informed consent for the study. The protocol and consent forms were approved by the institutional review boards at each site. Sample details are provided in supplementary material and table 1. DSM-IV diagnoses for all converters are given in supplementary table $\mathrm{S} 2$.

All participants (including both CHR and HC) received the Structured Clinical Interview for Diagnostic and Statistical Manual of Mental Disorders and the SIPS at baseline and at regular 6-month intervals up to approximately 24 months, and at the point of conversion to psychosis by trained clinicians. At each assessment point, prodromal symptom severity was quantified using the Scale of Prodromal Symptoms (SOPS). ${ }^{31}$

\section{fMRI Paradigm}

Subjects underwent fMRI scans while performing a paired-associate episodic memory task, ${ }^{33}$ including one run for encoding and another run for retrieval. During encoding, participants were presented a series of semantically unrelated word pairs for objects from 12 different categories (eg, animal, transportation, food) and colored picture pairs depicting each of the presented words. Participants were asked to remember the presented word pair by forming an association between them (eg, for the "rabbit-pail" pair: "put the rabbit in the pail"), and to press a button once the association had been made. Each pair was displayed for 4 seconds followed by a jittered interstimulus interval between 0.5 and 6 seconds. A baseline condition was included in which participants were presented with a series of 1-digit number pairs and colored squared pairs and were asked to sum up the 2 numbers. The session included 32 encoding trials and 24 active baseline trials lasting for 8.3 minutes ( 250 wholebrain volumes).

The retrieval session ensued directly after the encoding session. During retrieval, a pair of words was presented on the screen on each trial and subjects were asked to indicate whether the given word pair had been presented together during the encoding session by marking their confidence level (sure correct, maybe correct, sure incorrect, maybe incorrect). The word pairs during retrieval were either old pairs (50\% of trials) or reorganized new pairs using the words that had been shown during the encoding run $(50 \%$ of trials). During a baseline condition, participants were instructed to press the button corresponding to a confidence level simultaneously displayed on the screen. The retrieval session consisted of 64 retrieval trials and 16 baseline trials lasting for a 
Table 1. Demographic and Clinical Characteristics for the Baseline Sample in This Study

\begin{tabular}{|c|c|c|c|c|}
\hline & Converters $(n=18)$ & Nonconverters $(n=137)$ & Controls $(n=108)$ & $P$ Value \\
\hline \multicolumn{5}{|l|}{ Demographic data } \\
\hline Age (years) & $17.22 \pm 3.44$ & $19.01 \pm 4.19$ & $20.30 \pm 4.85$ & $\begin{array}{l}.01 \text { (overall) } \\
0.32(\mathrm{CHR}-\mathrm{C} \text { vs } \\
\text { CHR-NC) }\end{array}$ \\
\hline $\operatorname{Sex}(M / F)$ & $10 / 8$ & $81 / 56$ & $58 / 50$ & .65 \\
\hline Education (years) & $11.23 \pm 2.59$ & $11.58 \pm 2.60$ & $12.91 \pm 3.48$ & $\begin{array}{l}<.001 \text { (overall) } \\
.99(\text { CHR-C vs } \\
\text { CHR-NC) }\end{array}$ \\
\hline IQ & $110.28 \pm 12.37$ & $105.43 \pm 14.63$ & $110.99 \pm 14.29$ & $\begin{array}{l}.01 \text { (overall) } \\
.54(\mathrm{CHR}-\mathrm{C} \text { vs } \\
\text { CHR-NC) }\end{array}$ \\
\hline Site (no. of subjects) & $4 / 3 / 1 / 0 / 4 / 1 / 4 / 1$ & $17 / 35 / 15 / 14 / 11 / 12 / 29 / 4$ & $15 / 19 / 15 / 16 / 12 / 5 / 14 / 13$ & .08 (overall) \\
\hline \multicolumn{5}{|l|}{ Clinical data } \\
\hline SOPS-positive & $12.22 \pm 2.51$ & $11.41 \pm 3.83$ & $1.25 \pm 2.50$ & $\begin{array}{l}<.001 \text { (overall) } \\
.96(\text { CHR-C vs } \\
\text { CHR-NC) }\end{array}$ \\
\hline SOPS-negative & $9.83 \pm 6.35$ & $11.31 \pm 5.93$ & $1.46 \pm 2.03$ & $\begin{array}{l}<.001 \text { (overall) } \\
.65(\mathrm{CHR}-\mathrm{C} \text { vs } \\
\text { CHR-NC) }\end{array}$ \\
\hline SOPS-disorganization & $7.72 \pm 3.91$ & $5.24 \pm 3.23$ & $0.67 \pm 1.20$ & $\begin{array}{l}<.001 \text { (overall) } \\
.001(\text { CHR-C vs } \\
\text { CHR-NC) }\end{array}$ \\
\hline SOPS-general & $7.33 \pm 3.76$ & $8.76 \pm 4.43$ & $1.55 \pm 2.25$ & $\begin{array}{l}<.001 \text { (overall) } \\
.36(\text { CHR-C vs } \\
\text { CHR-NC) }\end{array}$ \\
\hline Antipsychotics (\% medicated) & 33 & 19 & 0 & $\begin{array}{l}<.001 \text { (overall) } \\
.16(\mathrm{CHR}-\mathrm{C} \text { vs } \\
\text { CHR-NC) }\end{array}$ \\
\hline $\begin{array}{l}\text { Antipsychotics } \\
\text { (chlorpromazine equivalent } \\
\text { dosage) }\end{array}$ & $107.08 \pm 175.22$ & $39.44 \pm 108.25$ & 0 & $\begin{array}{l}<.001 \text { (overall) } \\
.01(\mathrm{CHR}-\mathrm{C} \text { vs } \\
\text { CHR-NC) }\end{array}$ \\
\hline \multicolumn{5}{|l|}{ Cognitive data } \\
\hline $\begin{array}{l}\text { Hopkins verbal learning } \\
\text { test - revised }\end{array}$ & $24.17 \pm 6.45$ & $25.82 \pm 4.88$ & $28.15 \pm 4.11$ & $\begin{array}{l}<.001 \text { (overall) } \\
.49(\mathrm{CHR}-\mathrm{C} \text { vs } \\
\text { CHR-NC) }\end{array}$ \\
\hline $\begin{array}{l}\text { Brief visuospatial memory } \\
\text { test - revised }\end{array}$ & $23.72 \pm 7.49$ & $25.49 \pm 6.52$ & $28.57 \pm 4.68$ & $\begin{array}{l}<.001 \text { (overall) } \\
.71(\text { CHR-C vs } \\
\text { CHR-NC) }\end{array}$ \\
\hline \multicolumn{5}{|l|}{ Task data } \\
\hline Discrimination index $\left(d^{\prime}\right)$ & $1.77 \pm 0.93$ & $2.07 \pm 1.05$ & $2.44 \pm 0.96$ & $\begin{array}{l}.003 \text { (overall) } \\
.72 \text { (CHR-C vs } \\
\text { CHR-NC) }\end{array}$ \\
\hline Hit rate & $0.77 \pm 0.14$ & $0.81 \pm 0.12$ & $0.84 \pm 0.11$ & $\begin{array}{l}.02 \text { (overall) } \\
.69 \text { (CHR-C vs } \\
\text { CHR-NC) }\end{array}$ \\
\hline False alarm rate & $0.20 \pm 0.14$ & $0.18 \pm 0.18$ & $0.13 \pm 0.14$ & $\begin{array}{l}.03 \text { (overall) } \\
.99(\text { CHR-C vs } \\
\text { CHR-NC) }\end{array}$ \\
\hline Retrieval reaction time (ms) & $1993.41 \pm 278.21$ & $1957.39 \pm 260.84$ & $1968.58 \pm 235.03$ & .83 \\
\hline
\end{tabular}

Note: M, male; F, female; CHR-C, clinical high risk-converters; CHR-NC, clinical high risk-nonconverters; SOPS, Scale of Prodromal Symptoms; IQ, intelligence quotient.

total duration of 7.3 minutes (219 whole-brain volumes). A figure depicting the task is given in supplementary figure S1.

\section{Data Acquisition}

See supplementary methods for details on data acquisition.

\section{Behavioral Data Processing}

Task performance was evaluated based on signal detection theory. ${ }^{34}$ In particular, the discrimination index $(d)$ was used to assess the subjects' abilities to discriminate between old and reorganized new pairs. This was measured by the left-tail $z$ score of the hit rate $\left(Z_{\mathrm{Hit}}\right)$ minus the $z$ score of the false alarm rate $\left(Z_{\mathrm{FA}}\right)$. In particular, the "hit" 
trials (true positive) were defined as those with a positive response (ie, confidence levels at "sure correct" and "maybe correct") when old pairs were present, and the "false alarm" trials (false positive) were defined as those with a positive response when new pairs were present.

\section{fMRI Data Preprocessing}

fMRI data preprocessing followed that of previously published work ${ }^{35-37}$ using the standard procedures implemented in the Statistical Parametric Mapping software (SPM12; http://www.fil.ion.ucl.ac.uk/spm/software/ spm12/). In brief, all images were slice-time corrected, realigned for head motion, registered to the individual T1-weighted structural images, and spatially normalized to the Montreal Neurological Institute (MNI) template. Finally, the normalized images were spatially smoothed with an 8-mm full width at half-maximum of Gaussian kernel.

\section{Activation Analysis}

The preprocessed images were used for first-level analysis based on the general linear model, where task conditions, 24 head motion parameters (ie, the 6 rigid-body parameters generated from the realignment step, their first derivatives, and the squares of these 12 parameters ${ }^{38,39}$ ) and frame-wise displacement ${ }^{39,40}$ were modeled as regressors. The images were then high-pass filtered at $0.008 \mathrm{~Hz}$ and individual contrast maps (task condition vs baseline condition) were computed. The computed contrast images were further used for a second-level linear regression analysis, where group was modeled as the regressor of interest, and sex, age, and site were included as nuisance regressors. Notably, a recent study has demonstrated that linear regression with site as regressors can efficaciously and reliably remove site-related effects in imaging data. ${ }^{41}$ To control for type I error, significance was determined using family-wise error (FWE) correction across all voxels in the brain ${ }^{42}\left(N_{\text {voxels }}=145436\right)$.

\section{Network Analysis}

Network analysis followed previously published procedures. ${ }^{35-37}$ Here, we investigated 2 network measures that have been reported to be related to psychosis development in our previous work ${ }^{29}$ : network efficiency and node diversity. Node diversity was defined as the connectivity variance between a given node and all other nodes in the network, reflecting the heterogeneity of the connectivity pattern for that node. ${ }^{43}$ Network efficiency quantified the inverse of shortest path lengths in the network, which can be derived at both global and nodal levels. ${ }^{44}$ Details on network analysis are given in supplementary material.

Linear regression models were used to examine differences in the computed network measures between groups, where group, sex, age, site, and mean frame-wise displacement (ie, modeling head motion) were included as regressors. Network measures for each node were entered as dependent variables, and statistical significance was determined using FWE correction across all nodes in the brain network $\left(N_{\text {nodes }}=270\right)$.

\section{Association Analysis}

We further examined whether any observed alterations were related to task performance during scanning as well as clinical symptoms at baseline. For the observed activation differences, we extracted the first eigenvariates from 5-mm spheres around each of the peak coordinates in the group-level contrast map. These eigenvariates were further averaged across all spheres to generate a subject-specific activation metric. Pearson correlations were performed for each group between these subjectspecific metrics and performance and symptom variables. Here, task performance was measured using the $d$ ' scores and the mean reaction time during the retrieval session. Clinical symptoms were evaluated by the summed scores of each domain in the SOPS (positive, negative, disorganization, and general).

\section{Results}

\section{Task Performance}

No significant group differences were shown in selfreported response rate during encoding between outcome groups $(P=.07)$. There were significant differences in $Z_{\mathrm{Hit}}, Z_{\mathrm{FA}}$, and $d$ ' scores by outcome group $(P<.02$; table 1), with both converters and nonconverters showing lower $d$ ' scores compared with controls $\left(P_{\text {Bonferroni }}<\right.$ .03 ); however, converters and nonconverters did not differ from each other. In contrast, there were no significant group differences in retrieval response time $(P=.83)$.

\section{Activation Alterations at the Group Level}

When controlling for multiple comparisons voxel-wise throughout the brain, there were no significant group differences in activation during encoding. However, during retrieval, a significant group effect on brain activation was observed in 5 brain regions (figure 1A; see supplementary table S1 for coordinates in the MNI space): left superior frontal gyrus (SFG) $\left(T_{252}=4.75, P_{\mathrm{FWE}}=.034\right)$, left inferior frontal gyrus (IFG) $\left(T_{252}=4.99, P_{\mathrm{FWE}}=.013\right)$, left inferior parietal lobule (IPL) $\left(T_{252}=4.73, P_{\mathrm{FWE}}=.035\right)$, left superior temporal gyrus $\left(T_{252}=5.71, P_{\mathrm{FWE}}=.001\right)$, and right middle temporal gyrus $\left(T_{252}=4.89, P_{\mathrm{FWE}}=.019\right)$. In each region, subjects at $\mathrm{CHR}$ showed significantly higher activation compared with controls, with converters showing significantly higher activation than nonconverters (figure 1B). Secondary analyses showed that the detected effects were presented for both correct trials and incorrect trials, and for both old word pairs and new word pairs (see supplementary results), suggesting that hyperactivation 

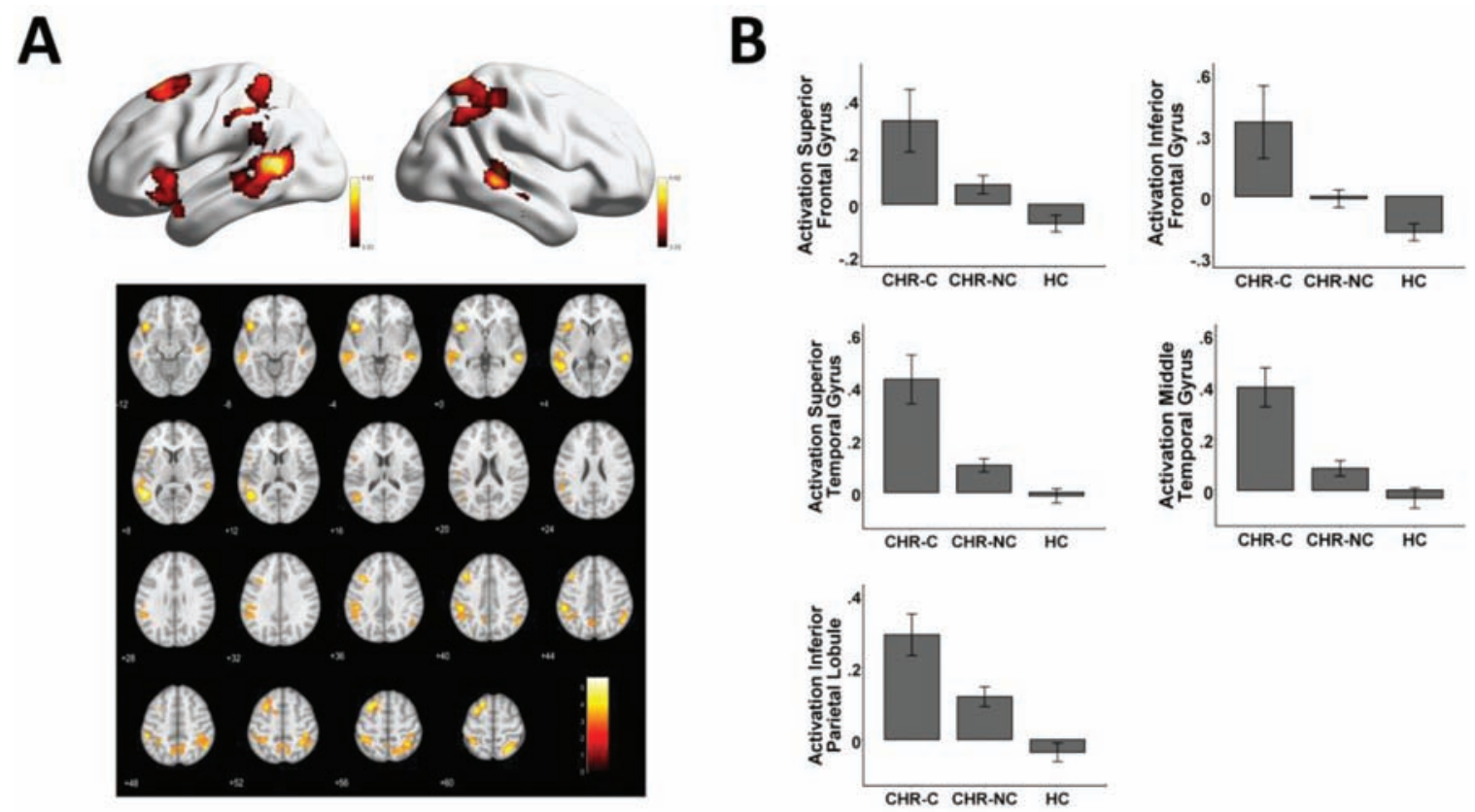

Fig. 1. Between-group activation differences during memory retrieval at baseline scan (panel A). Subjects at CHR showed significantly higher activation in the prefrontal, parietal and temporal cortices compared with controls, with hyperactivation more prominent in converters than nonconverters. For presentation purposes, the activation maps are thresholded at $P<.001$ with cluster size of more than 300 voxels. The mean first eigenvariates across all voxels in each region are plotted in panel $\mathrm{B}$, with error bars indicating the standard error. CHR, clinical high risk; CHR-C, clinical high risk-converters; CHR-NC, clinical high risk-nonconverters; HC, healthy control.

during retrieval is a robust finding not driven by a single trial type in the experiment.

\section{Network Alterations at the Group Level}

The network analysis did not reveal any significant differences between groups in terms of network efficiency and network diversity at the global nor nodal levels $\left(P_{\mathrm{FWE}}>.27\right)$.

\section{Associations With Antipsychotic Medications}

To determine whether the observed activation findings during retrieval were affected by antipsychotic medications, we extracted mean activation metrics from these 5 regions and compared these metrics between converters and nonconverters separately for unmedicated and medicated participants. The detected activation differences were in fact more pronounced among unmedicated subjects than in medicated subjects (figure 2). Specifically, unmedicated converters had significantly higher activation in these regions than unmedicated nonconverters $(P<.001)$, whereas no significant differences were found between medicated converters and medicated nonconverters $(P=.21)$, suggesting that the pattern of hyperactivation during memory retrieval is not explained by antipsychotic medication.

\section{Associations With Performance Measures and Symptom Severity}

There were no significant correlations of activation metrics with $d$ ' scores nor with encoding response rate during

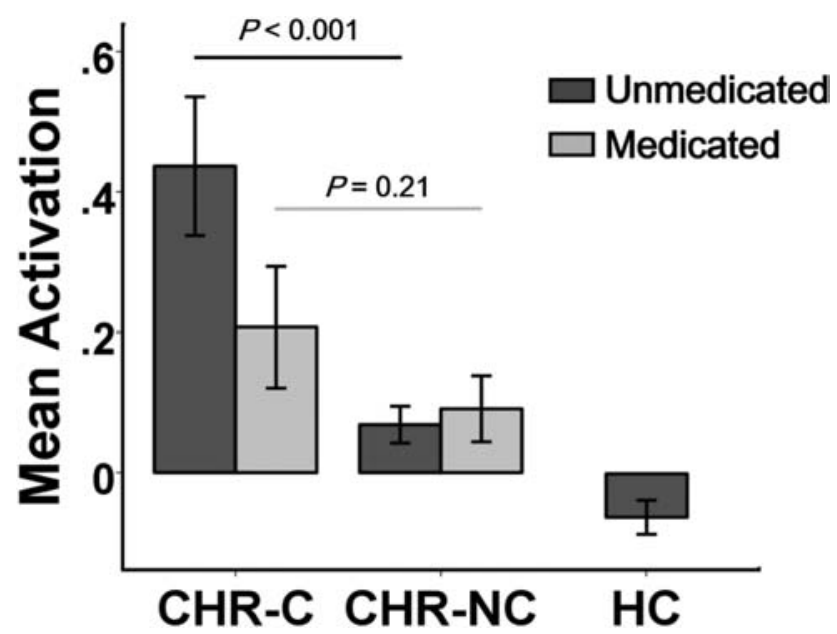

Fig. 2. Mean activation differences between groups during memory retrieval separated by medication status. Significant differences were shown between unmedicated converters and unmedicated nonconverters, but not between medicated converters and medicated nonconverters. The y-axis shows the mean first eigenvariates across all identified regions, with error bars indicating the standard error. $\mathrm{CHR}-\mathrm{C}$, clinical high riskconverters; CHR-NC, clinical high risk-nonconverters; HC, healthy control.

scan in any of the 3 groups separately $(P>.16)$. However, significant positive correlations were found between activation metrics and retrieval reaction time in converters ( $R=0.61, P=.009$, figure 3 ), but not in nonconverters and controls. No significant associations were found 


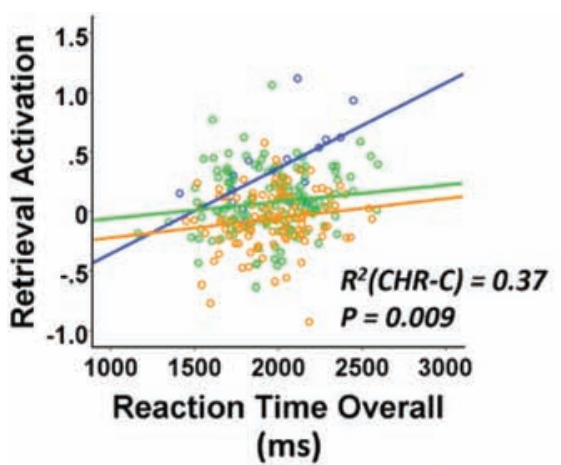

(ms)
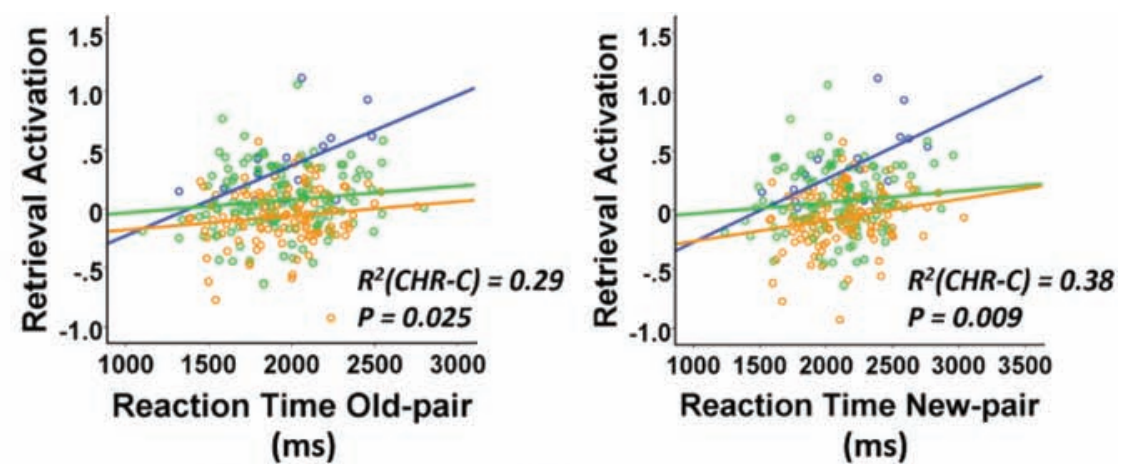

O CHR-C OCHR-NC O HC

Fig. 3. Association of activation alterations with task performance. Mean activation measures were significantly correlated with task reaction time during memory retrieval in converters. The y-axis shows the mean first eigenvariates across all identified regions, $\mathrm{CHR}-\mathrm{C}$, clinical high risk-converters; CHR-NC, clinical high risk-nonconverters; $\mathrm{HC}$, healthy control.

between the activation metrics and symptom severity in any of the groups.

\section{Individual-Level Prediction Analysis}

On the basis of these findings, we performed a secondary multivariate receiver operating characteristic (ROC) curve analysis for a logistic regression model to assess individual-level predictability of the activation measures for psychosis. Due to the lack of an independent test sample, we used independent anatomical masks to extract activation-based predictors to minimize potential circularity in the modeling. Here, 5 independent anatomical masks (ie, left SFG, left IFG, left IPL, left superior temporal gyrus, and right middle temporal gyrus) defined by the automated anatomical labeling (AAL) brain atlas ${ }^{45}$ were selected for the analysis based on the group-level activation findings during retrieval. The first eigenvariates for each of the 5 masks were extracted from individuals' contrast maps and were included in the model as predictors. The ROC curves were plotted to illustrate the accuracy of these predictors in distinguishing converters from $\mathrm{CHR}$ and from both CHR and controls. The areas under curve (AUCs) were subsequently calculated as the index of accuracy of the prediction models. To test the significance of the derived AUCs, we randomized the sample by 10000 permutations, where group labels were randomly reshuffled for each subject during each permutation. Empirical $P$ values were determined as the proportion of the resampled AUCs in the permutation distribution that were larger than the AUC in the original sample.

The ROC curves using baseline retrieval-related activation measures to distinguish converters from nonconverters and controls are presented in figure 4. The model for predicting converters among the CHR subjects obtained an AUC of $0.768(P=.009)$, and the model for predicting converters among both CHR subjects and controls had an AUC of $0.773(P=.005)$.

\section{Discussion}

Using data from the NAPLS-2 consortium, we found evidence for memory-associated functional brain alterations in subjects at $\mathrm{CHR}$ for psychosis. In particular, CHR individuals showed increased activation during retrieval at baseline compared with controls, a pattern that was more pronounced in individuals who converted to psychosis and was independent of exposure to antipsychotic drugs and performance differences on the underlying memory task.

Five regions were associated with hyperactivation during retrieval in subjects at CHR and in particular among converters: SFG, IFG, IPL, and lateral temporal cortices (LTCs) in both hemispheres. Interestingly, despite inconsistent directions reported in the literature, which may relate to the heterogeneity of samples and tasks used, altered retrieval activation in all of these regions has been found in patients with schizophrenia ${ }^{3,15,19,46-48}$ and has further been summarized in several meta-analyses assessing episodic memory processing in schizophrenia. ${ }^{14,16}$ Here, our results extend prior findings to a sample of CHR subjects before the onset of psychotic disorders. These observations together suggest activation abnormalities in the prefrontal, parietal, and temporal cortices as a biological trait underlying memory retrieval deficits during both the prodromal phase and fully psychotic state. Notably, the observed differences could be robustly detected in subjects who did not receive any antipsychotic medication, indicating that these activation changes are not a secondary phenomenon of clinical treatment but rather reflect neural deficits related to psychosis risk. Although we did not observe a significant difference between converters and nonconverters with antipsychotic medication, which might suggest that antipsychotics have potential to attenuate the hyperactivation in these regions, great caution is warranted in interpreting this result given the small sample size of medicated CHR subjects. 

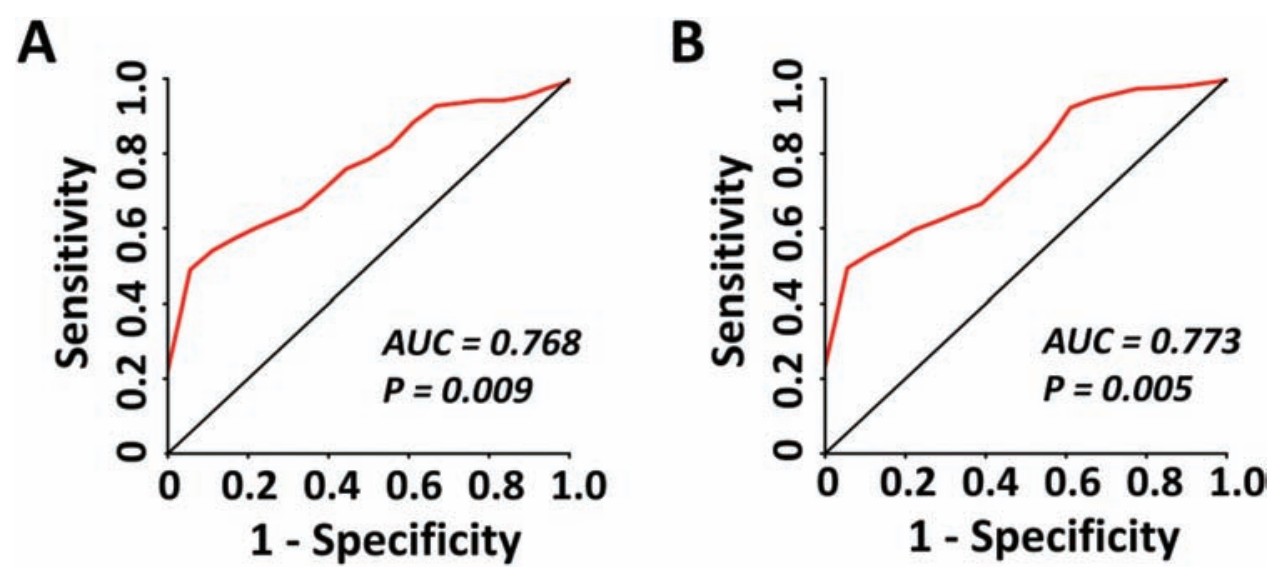

Fig. 4. The multivariate receiver operating characteristic curves for baseline activation measures showed an area under curve (AUC) of 0.768 for distinguishing converters from nonconverters among subjects at clinical high risk (panel A), and an AUC of 0.773 for distinguishing converters from both nonconverters and controls (panel B). For both curves, the AUCs were highly significant from 10000 permutations, indicating better prediction accuracy than chance.

The detected hyperactivation was exclusively in regions that are crucial for associative and episodic memory processing in humans. Specifically, the SFG as part of the DLPFC has been considered to be involved in cognitive control mechanisms for information retrieval, particularly postretrieval monitoring. ${ }^{49,50}$ The IFG as part of the VLPFC is a key region for the modulation of information input into the memory system and the maintenance of successfully retrieved information..$^{51,52}$ The IPL is related to attention engagement and sense of familiarity during retrieval, ${ }^{53,54}$ and the LTC may play a key role in semantic processing during encoding and retrieval of word pairs. ${ }^{55,56}$ Together, the conjoint hyperactivation of these regions may imply that excessive control is needed for successful information retrieval in subjects at $\mathrm{CHR}$ and particular those who will convert to full psychosis. This interpretation is supported by the fact that higher activation in these regions was correlated with longer memory retrieval time but not discrimination ability in converters, a pattern suggestive of greater retrieval effort. The increased effort, in turn, may involve more task-related attention and executive control during the retrieval process and lead to enhanced activation of the aforementioned regions in converters. ${ }^{57,58}$ As a result, these findings suggest that hyperactivation of memory retrieval regions, possibly attributed to increased effortful processing during retrieval testing, marks processes associated with conversion to psychosis. The secondary analyses showing hyperactivation pattern for both correct and incorrect trials and for both old and new word pairs further support this argument.

In contrast to the significant findings in brain activation, brain network analysis did not reveal any significant results in network efficiency nor network diversity in subjects at CHR. This is consistent with our previous work that resting-state functional network configuration disrupts gradually in tandem with the progression of psychosis but is intact at the baseline scan. ${ }^{29}$ Although we do not have a sufficient sample tracking the longitudinal change of brain networks during the memory task, the reported baseline findings may suggest that measures of network efficiency and diversity may serve as biomarkers for tracking the development of psychosis rather than biomarkers for predicting psychosis at the early stage of prodromal syndromes. Taken together, it seems that abnormal brain activity may occur before overt network architecture changes in subjects at CHR for psychosis.

Our study has several limitations. First, the sample size for converters was relatively small and the conversion rate among subjects with fMRI data available was relatively low $(\sim 12 \%)$, thus further replications of the present findings are encouraged using larger sample sizes. Second, as presented in table 1, although demographic variables were matched between converters and nonconverters, the clinical groups differed from controls on some measures. Third, the ROC analysis was not derived from an independent sample. Although we sought to mitigate the effect of this limitation by using anatomical masks that were independent of group-level activation clusters, the resulting AUCs are nevertheless still likely to be overestimated to a certain degree. Fourth, because all participants had their clinical assessments up to approximately 24 months, it is possible that the nonconverter group contains some subjects who might eventually convert to psychosis after the last assessment. However, as previously discussed, ${ }^{59}$ this possibility would actually attenuate the differences between the converter and nonconverter groups, suggesting that the observed effects in this study may be underestimated. Fifth, the detected findings in this study were based on a specific paired-associate memory paradigm and may not necessarily generalize to all tasks used to challenge memory functions in psychosis studies. In particular, the paradigm used in this study 
used both verbal (words) and nonverbal (pictures) stimuli during the encoding phase but only verbal stimuli (words) during the retrieval phase. Although including pictures would help participants encode presented word pairs, this design nevertheless introduced additional experimental components that were not exactly matched to the retrieval phase. This discrepancy might to certain degree contribute to the differential activation outcomes between the 2 phases, because impairments of verbal and visual memories might be different in patients with schizophrenia. ${ }^{60,61}$ Finally, the memory deficits in converters may reflect difficulty in recalling the encoded information at retrieval, difficulty in creating the associations during encoding, or both. Although these 2 factors cannot be completely isolated in a memory task as used here, we did not observe a significant between-group difference in self-reported encoding response rate, nor a significant correlation between retrieval hyperactivation and encoding response rate, suggesting that activation differences at retrieval are unlikely to be caused purely by failure in forming an association at encoding.

\section{Conclusion}

In summary, this study provides first evidence for functional alterations during associative memory processing in individuals at CHR for psychosis. Our findings support increased baseline retrieval activation as a potential biomarker for psychosis prediction. Further work is encouraged to replicate these findings and to test the predictive power of these findings in independent cohorts.

\section{Supplementary Material}

Supplementary data are available at Schizophrenia Bulletin online.

\section{Funding}

This work was supported by gifts from the Staglin Music Festival for Mental Health and International Mental Health Research Organization and by National Institute of Health (NIH) grants (U01 MH081902 to TDC, P50 MH066286 and the Miller Family Endowed Term Chair to CEB, U01 MH081857 to BAC, U01 MH82022 to SWW, U01 MH066134 to JA, U01 MH081944 to KSC, R01 U01 MH066069 to DOP, R01 MH076989 to DHM, U01 MH081928 to LJS, and U01 MH081988 to EFW).

\section{Acknowledgments}

Dr Cannon has served as a consultant for BoehringerIngelheim Pharmaceuticals and Lundbeck A/S. The other authors report no conflicts of interest.

\section{References}

1. Barch DM, Sheffield JM. Cognitive impairments in psychotic disorders: common mechanisms and measurement. World Psychiatry. 2014;13:224-232.

2. Achim AM, Lepage M. Is associative recognition more impaired than item recognition memory in schizophrenia? A meta-analysis. Brain Cogn. 2003;53:121-124.

3. Lepage M, Montoya A, Pelletier M, Achim AM, Menear M, Lal S. Associative memory encoding and recognition in schizophrenia: an event-related fMRI study. Biol Psychiatry. 2006;60:1215-1223.

4. Keshavan MS, Kulkarni S, Bhojraj T, et al. Premorbid cognitive deficits in young relatives of schizophrenia patients. Front Hum Neurosci. 2010;3:62.

5. Broome MR, Fusar-Poli P, Matthiasson P, et al. Neural correlates of visuospatial working memory in the 'at-risk mental state'. Psychol Med. 2010;40:1987-1999.

6. Haut KM, van Erp TG, Knowlton B, et al. Contributions of feature binding during encoding and functional connectivity of the medial temporal lobe structures to episodic memory deficits across the prodromal and first-episode phases of schizophrenia. Clin Psychol Sci. 2015;3:159-174.

7. Seidman LJ, Shapiro DI, Stone WS, et al. Association of neurocognition with transition to psychosis: baseline functioning in the second phase of the North American Prodrome Longitudinal Study. JAMA Psychiatry. 2016;73:1239-1248.

8. Fusar-Poli P, Deste G, Smieskova R, et al. Cognitive functioning in prodromal psychosis: a meta-analysis. Arch Gen Psychiatry. 2012;69:562-571.

9. Spaniol J, Davidson PS, Kim AS, Han H, Moscovitch M, Grady CL. Event-related fMRI studies of episodic encoding and retrieval: meta-analyses using activation likelihood estimation. Neuropsychologia. 2009;47:1765-1779.

10. Kirwan CB, Stark CE. Medial temporal lobe activation during encoding and retrieval of novel face-name pairs. Hippocampus. 2004;14:919-930.

11. Blumenfeld RS, Parks CM, Yonelinas AP, Ranganath C. Putting the pieces together: the role of dorsolateral prefrontal cortex in relational memory encoding. J Cogn Neurosci. 2011;23:257-265.

12. Park H, Rugg MD. Neural correlates of successful encoding of semantically and phonologically mediated inter-item associations. Neuroimage. 2008;43:165-172.

13. Woodcock EA, Wadehra S, Diwadkar VA. Network profiles of the dorsal anterior cingulate and dorsal prefrontal cortex in schizophrenia during hippocampal-based associative memory. Front Syst Neurosci. 2016;10:32.

14. Achim AM, Lepage M. Episodic memory-related activation in schizophrenia: meta-analysis. $\mathrm{Br} J$ Psychiatry. 2005;187:500-509.

15. Ragland JD, Gur RC, Valdez J, et al. Event-related fMRI of frontotemporal activity during word encoding and recognition in schizophrenia. Am J Psychiatry. 2004;161:1004-1015.

16. Ragland JD, Laird AR, Ranganath C, Blumenfeld RS, Gonzales SM, Glahn DC. Prefrontal activation deficits during episodic memory in schizophrenia. Am J Psychiatry. 2009; 166:863-874.

17. Francis MM, Hummer TA, Vohs JL, et al. Functional neuroanatomical correlates of episodic memory impairment in early phase psychosis. Brain Imaging Behav. 2016;10:1-11.

18. Pirnia T, Woods RP, Hamilton LS, et al. Hippocampal dysfunction during declarative memory encoding in 
schizophrenia and effects of genetic liability. Schizophr Res. 2015;161:357-366.

19. Ongür D, Cullen TJ, Wolf DH, et al. The neural basis of relational memory deficits in schizophrenia. Arch Gen Psychiatry. 2006;63:356-365.

20. Ragland JD, Ranganath C, Harms MP, et al. Functional and neuroanatomic specificity of episodic memory dysfunction in schizophrenia: a functional magnetic resonance imaging study of the relational and item-specific encoding task. JAMA Psychiatry. 2015;72:909-916.

21. Ray KL, Lesh TA, Howell AM, et al. Functional network changes and cognitive control in schizophrenia. Neuroimage Clin. 2017;15:161-170.

22. Avery SN, Rogers BP, Heckers S. Hippocampal network modularity is associated with relational memory dysfunction in schizophrenia. Biol Psychiatry Cogn Neurosci Neuroimaging. 2018;3:423-432.

23. Hutcheson NL, Sreenivasan KR, Deshpande G, et al. Effective connectivity during episodic memory retrieval in schizophrenia participants before and after antipsychotic medication. Hum Brain Mapp. 2015;36:1442-1457.

24. Di Giorgio A, Gelao B, Caforio G, et al. Evidence that hippocampal-parahippocampal dysfunction is related to genetic risk for schizophrenia. Psychol Med. 2013;43:1661-1671.

25. Rasetti R, Mattay VS, White MG, et al. Altered hippocampal-parahippocampal function during stimulus encoding: a potential indicator of genetic liability for schizophrenia. JAMA Psychiatry. 2014;71:236-247.

26. Erk S, Meyer-Lindenberg A, Schmierer P, et al. Hippocampal and frontolimbic function as intermediate phenotype for psychosis: evidence from healthy relatives and a common risk variant in CACNA1C. Biol Psychiatry. 2014;76:466-475.

27. Stolz E, Pancholi KM, Goradia DD, et al. Brain activation patterns during visual episodic memory processing among first-degree relatives of schizophrenia subjects. Neuroimage. 2012;63:1154-1161.

28. Addington J, Cadenhead KS, Cornblatt BA, et al. North American Prodrome Longitudinal Study (NAPLS 2): overview and recruitment. Schizophr Res. 2012;142:77-82.

29. Cao H, Chung Y, McEwen S, et al. Progressive reconfiguration of resting-state brain networks as psychosis develops: preliminary results from the North American Prodrome Longitudinal Study (NAPLS) consortium. bioRxiv. August 21, 2017; doi: https://doi.org/10.1101/179242.

30. Wechsler D. Wechsler Abbreviated Scale of Intelligence. New York, NY: Psychological Corporation; 1999.

31. McGlashan TH, Miller TJ, Woods SW, Hoffman RE, Davidson L. Instrument for the assessment of prodromal symptoms and states. In: Miller T, Mednick SA, McGlashan $\mathrm{TH}$, Libiger J, Johannessen JO, eds. Early Intervention in Psychotic Disorders. Dordrecht: Springer Netherlands; 2001:135-149.

32. First MB, Spitzer RL, Gibbon ML, Williams JBW. Structured Clinical Interview for DSM-IV-TR Axis I Disorders, Research Version, Non-Patient Edition (SCID-I/P). New York: Biometrics Research, New York State Psychiatric Institute; 2002.

33. Cao H, McEwen SC, Forsyth JK, et al. Toward leveraging human connectomic data in large consortia: generalizability of fMRI-based brain graphs across sites, sessions, and paradigms. Cereb Cortex. 2018. doi:10.1093/cercor/bhy032

34. Snodgrass JG, Corwin J. Pragmatics of measuring recognition memory: applications to dementia and amnesia. $J$ Exp Psychol Gen. 1988;117:34-50.
35. Cao H, Plichta MM, Schäfer A, et al. Test-retest reliability of fMRI-based graph theoretical properties during working memory, emotion processing, and resting state. Neuroimage. 2014;84:888-900.

36. Cao H, Bertolino A, Walter $\mathrm{H}$, et al. Altered functional subnetwork during emotional face processing: a potential intermediate phenotype for schizophrenia. JAMA Psychiatry. 2016;73:598-605.

37. Cao H, Harneit A, Walter $\mathrm{H}$, et al. The 5-HTTLPR polymorphism affects network-based functional connectivity in the visual-limbic system in healthy adults. Neuropsychopharmacology. 2018;43:406-414.

38. Satterthwaite TD, Elliott MA, Gerraty RT, et al. An improved framework for confound regression and filtering for control of motion artifact in the preprocessing of resting-state functional connectivity data. Neuroimage. 2013;64:240-256.

39. Power JD, Mitra A, Laumann TO, Snyder AZ, Schlaggar BL, Petersen SE. Methods to detect, characterize, and remove motion artifact in resting state fMRI. Neuroimage. 2014;84:320-341.

40. Power JD, Barnes KA, Snyder AZ, Schlaggar BL, Petersen SE. Spurious but systematic correlations in functional connectivity MRI networks arise from subject motion. Neuroimage. 2012;59:2142-2154.

41. Fortin JP, Cullen N, Sheline YI, et al. Harmonization of cortical thickness measurements across scanners and sites. Neuroimage. 2018;167:104-120.

42. Penny WD, Friston KJ, Ashburner JT, Kiebel SJ, Nichols TE. Statistical Parametric Mapping: The Analysis of Functional Brain Images. Cambridge, MA: Academic Press; 2006.

43. Lynall ME, Bassett DS, Kerwin R, et al. Functional connectivity and brain networks in schizophrenia. J Neurosci. 2010;30:9477-9487.

44. Rubinov M, Sporns O. Complex network measures of brain connectivity: uses and interpretations. Neuroimage. 2010;52:1059-1069.

45. Tzourio-Mazoyer N, Landeau B, Papathanassiou D, et al. Automated anatomical labeling of activations in SPM using a macroscopic anatomical parcellation of the MNI MRI single-subject brain. Neuroimage. 2002;15:273-289.

46. Ragland JD, Gur RC, Valdez JN, et al. Levels-of-processing effect on frontotemporal function in schizophrenia during word encoding and recognition. Am J Psychiatry. 2005;162:1840-1848.

47. Heckers S, Rauch SL, Goff D, et al. Impaired recruitment of the hippocampus during conscious recollection in schizophrenia. Nat Neurosci. 1998;1:318-323.

48. Heckers S, Curran T, Goff D, et al. Abnormalities in the thalamus and prefrontal cortex during episodic object recognition in schizophrenia. Biol Psychiatry. 2000;48:651-657.

49. Achim AM, Lepage M. Dorsolateral prefrontal cortex involvement in memory post-retrieval monitoring revealed in both item and associative recognition tests. Neuroimage. 2005;24:1113-1121.

50. Rugg MD, Henson RN, Robb WG. Neural correlates of retrieval processing in the prefrontal cortex during recognition and exclusion tasks. Neuropsychologia. 2003;41:40-52.

51. Simons JS, Spiers HJ. Prefrontal and medial temporal lobe interactions in long-term memory. Nat Rev Neurosci. 2003;4:637-648.

52. Barredo J, Öztekin I, Badre D. Ventral fronto-temporal pathway supporting cognitive control of episodic memory retrieval. Cereb Cortex. 2015;25:1004-1019. 
53. Hutchinson JB, Uncapher MR, Weiner KS, et al. Functional heterogeneity in posterior parietal cortex across attention and episodic memory retrieval. Cereb Cortex. 2014;24:49-66.

54. Sestieri C, Shulman GL, Corbetta M. The contribution of the human posterior parietal cortex to episodic memory. Nat Rev Neurosci. 2017;18:183-192.

55. Ojemann GA, Creutzfeldt O, Lettich E, Haglund MM. Neuronal activity in human lateral temporal cortex related to short-term verbal memory, naming and reading. Brain. 1988;111 (Pt 6):1383-1403.

56. Martin A, Chao LL. Semantic memory and the brain: structure and processes. Curr Opin Neurobiol. 2001;11:194-201.

57. Shimamura AP. Memory Retrieval and Executive Control. Principles of Frontal Lobe Function. Oxford: Oxford University Press; 2002:210-220.
58. Levy BJ, Anderson MC. Inhibitory processes and the control of memory retrieval. Trends Cogn Sci. 2002;6:299-305.

59. Anticevic A, Haut K, Murray JD, et al. Association of thalamic dysconnectivity and conversion to psychosis in youth and young adults at elevated clinical risk. JAMA Psychiatry. 2015;72:882-891.

60. Bruder GE, Wexler BE, Sage MM, Gil RB, Gorman JM. Verbal memory in schizophrenia: additional evidence of subtypes having different cognitive deficits. Schizophr Res. 2004;68:137-147.

61. Skelley SL, Goldberg TE, Egan MF, Weinberger DR, Gold JM. Verbal and visual memory: characterizing the clinical and intermediate phenotype in schizophrenia. Schizophr Res. 2008;105:78-85. 